Pacific Journal of Mathematics

INTEGRALITY OF SUBRINGS OF MATRIX RING 


\title{
INTEGRALITY OF SUBRINGS OF MATRIX RINGS
}

\author{
LANCE W. SMall AND AdRian R. WADSWORTH
}

\begin{abstract}
Let $A \subseteq B$ be commutative rings, and $\Gamma$ a multiplicative monoid which generates the matrix ring $M_{n}(B)$ as a $B$-module. Suppose that for each $\gamma \in \Gamma$ its trace $\operatorname{tr}(\gamma)$ is integral over $A$. We will show that if $A$ is an algebra over the rational numbers or if for every prime ideal $P$ of $A$, the integral closure of $A / P$ is completely integrally closed, then the algebra $A(\Gamma)$ generated by $\Gamma$ over $A$ is integral over $A$. This generalizes a theorem of Bass which says that if $A$ is Noetherian (and the trace condition holds), then $A(\Gamma)$ is a finitely generated $A$-module.
\end{abstract}

Our generalizations of the theorem of Bass [B, Th. 3.3] yield a simplified proof of that theorem. Bass's proof used techniques of Procesi in [P, Ch. VI] and involved completion and faithfully flat descent. The arguments given here are based on elementary properties of integral closure and complete integral closure. They serve also to illuminate a couple of theorems of A. Braun concerning prime p.i. rings integral over the center.

One might expect that integrality of $\operatorname{tr}(\gamma)$ for $\gamma \in \Gamma$ would be sufficient to assure that $A(\Gamma)$ is integral over $A$. But this is not so, as we will show with a counterexample. As it frequently happens with traces, complications arise in prime characteristic.

1. Integrality and complete integral closure. Recall that if $A$ is an integral domain and $b$ lies in its quotient field, $b$ is said to be almost integral over $A$ if there is an $a \in A, a \neq 0$, such that $a b^{i} \in A$ for all integers $i \geq 1$. $A$ is said to be completely integrally closed (c.i.c.) if every element almost integral over $A$ lies in $A$. Recall that a Krull domain is completely integrally closed [Bo, $§ 1$, No. 3], as indeed is any intersection of rank 1 valuation rings. (However, examples are known of c.i.c. domains which are not intersections of rank 1 valuation rings - see [Nk] or [G, App. 4].) If $A$ is a Noetherian domain, the Mori-Nagata Theorem [N, (33.10)] says that the integral closure of $A$ is a Krull domain, hence is c.i.c.

LEMMA 1. Let $A$ be a completely integrally closed integral domain with quotient field $F$, and let $B$ be the integral closure of $A$ in any extension field of $F$. Then $B$ is completely integrally closed. 
Proof. This is [K, Satz 11].

LEMMA 2. Let $R$ be a ring and $A$ a subring of the center of $R$, such that $A$ contains no zero divisors of $R$. Suppose the integral closure of $A$ is completely integrally closed. If there is an $a \in A, a \neq 0$, with aR integral over $A$, then $R$ is integral over $A$.

Proof. If not, take $t \in R$ with $t$ not integral over $A$. We may assume $R=A[t]$, which is commutative. Let $S=\{b \cdot f(t) \mid b \in A, b \neq 0$ and $f \in A[x], f$ monic $\}$, a multiplicatively closed subset of $R$ not containing 0 . Let $P$ be an ideal of $R$ maximal such that $P \cap S=\varnothing$. Then $P \cap A=(0)$ and, replacing $R$ by $R / P$, we may assume that $R$ is an integral domain. Let $B$ be the integral closure of $A$ in the quotient field of $R$. By hypothesis $a R \subseteq B$; hence, $t$ is almost integral over $B$. By Lemma $1, t \in B$, contradicting the choice of $t$.

Here is a variant of Lemma 2. It is proved in the same way, but using $S=\left\{a^{i} f(t) \mid f \in A[x], f\right.$ monic $\}$ and applying the Mori-Nagata Theorem.

LEMMA 2 '. Let $A$ be a Noetherian subring of the center of a ring $R$; let $a \in A$ be a regular element of $R$. If aR is integral over $A$, then $R$ is integral over $A$.

These lemmas can be applied to prime p.i. rings, yielding short proofs of one theorem of A. Braun and part of another. For, if $R$ is a prime p.i. ring with center $C$, then a theorem of Amitsur using central polynomials [A, Th. 6] says that there is a $\delta \in C, \delta \neq 0$, such that $\delta R$ lies in a ring which is a free $C$-module of finite rank. It follows by the usual determinant argument that $\delta R$ is integral over $C$.

Proposition 3 (Braun, $\left[\mathbf{B r}_{1}\right.$, Th. 2.7]). Let $R$ be a prime p.i. ring which is finitely-generated as an algebra over some commutative Noetherian ring $A$. Let $C$ be the center of $R$. Then $R$ is a finitely-generated $C$-module if and only if the integral closure of $C$ is a Krull domain.

Proof. If $R$ is a finitely-generated $C$-module, then by the Artin-Tate Lemma [AT] $C$ is a finitely-generated $A$-algebra. Hence, $C$ is Noetherian, so by the Mori-Nagata Theorem its integral closure is a Krull domain. Conversely, suppose the integral closure of $C$ is a Krull domain (hence completely integrally closed). By Lemma 2 and the remarks above, $R$ is 
integral over $C$. Then, by a theorem of Procesi $[\mathbf{P}, \mathrm{p} .128], R$ is a finitely generated $C$-module.

Proposition 4 (Braun [Br $\mathbf{B r}_{2}$,pp. 13-14], Schelter [S, Cor. 2 to Th. 2]). If $R$ is a prime p.i. ring with center $C$, and if the integral closure of $C$ is completely integrally closed, then $R$ is integral over $C$.

Proof. Apply Lemma 2 and the remarks preceding Prop. 3.

2. Integrality when traces are integral. We now return to Bass's theorem. Throughout this section, let $A \subseteq B$ be commutative rings, and $\Gamma$ a multiplicative monoid in the $n \times n$ matrix ring $M_{n}(B)$ which generates $M_{n}(B)$ as a $B$-module. Let $A(\Gamma)$ be the $A$-module (and algebra) generated by $\Gamma$. We wish to consider when the following statement is true:

If $\operatorname{tr}(\gamma)$ is integral over $A$, for each $\gamma \in \Gamma$, then $A(\Gamma)$ is integral over $A$.

Proposition 5. If $A$ is an algebra over a field $F$, and if char $F=0$ or $\operatorname{char} F=p>n$, then $(*)$ is true.

Proof. Consider first the generic $n \times n$ matrix $\alpha$, whose entries are the commuting indeterminates $x_{11}, x_{12}, \ldots, x_{n n}$. Let $\lambda_{1}, \ldots, \lambda_{n}$ be the eigenvalues of $\alpha$ in an algebraic closure of $F\left(x_{11}, \ldots, x_{n n}\right)$, and let the characteristic polynomial of $\alpha$ be

$$
\chi_{\alpha}(x)=x^{n}+c_{1} x^{n-1}+\cdots+c_{n} .
$$

For each $i$, let $t_{i}=\operatorname{tr}\left(\alpha^{l}\right)=\lambda_{1}^{i}+\cdots+\lambda_{n}^{i}$; these traces are related to the $c_{j}$ 's by Newton's identities (see, e.g., [C, pp. 436-437], or [H, p. 249]):

$$
t_{i}+\sum_{j=1}^{i-1} c_{j} t_{i-j}+i c_{i}=0, \quad 1 \leq i \leq n .
$$

Now, take any $\gamma \in A(\Gamma)$. Then $\operatorname{tr}(\gamma)$ is integral over $A$, since $\gamma$ is an $A$-linear combination of elements of $\Gamma$. Specializing from $\alpha$ to $\gamma$ we obtain formulas corresponding to (1) relating the traces $\operatorname{tr}\left(\gamma^{l}\right)$ and the coefficients of the characteristic polynomial $\chi_{\gamma}(x)$. The assumption on char $F$ assures that we can divide by $2,3, \ldots, n$. Therefore, we may solve recursively for the $c_{i}$ in (1), obtaining expressions for the coefficients of $\chi_{\gamma}(x)$ as polynomials in $\left\{\operatorname{tr}\left(\gamma^{i}\right) \mid 1 \leq i \leq n\right\}$. Thus, the coefficients of $\chi_{\gamma}(x)$ are integral over $A$; hence $\gamma$ is integral over $A$, as desired. 
Remarks. The argument for Prop. 5 is valid for any ring $A$ in which the images of $2,3, \ldots, n$ are all units. Note also that the assumption that $B(\Gamma)=M_{n}(B)$ was not used.

Proposition 6. Suppose that for every prime ideal $P$ of $A$, the integral closure of $A / P$ is completely integrally closed. Then $(*)$ is true.

Proof. If not, take any $t \in A(\Gamma), t$ not integral over $A$. Let $S=$ $\{f(t) \mid f \in A[x], f$ monic $\} \subseteq M_{n}(B) . S$ is closed under multiplication and $0 \notin S$. Let $Q$ be an ideal of $M_{n}(B)$, maximal with the property that $Q \cap S=\varnothing$. Then $Q$ is a prime ideal and, reducing $\bmod Q$, we may assume that $A$ and $B$ are integral domains. Furthermore, since there is no harm in enlarging $B$ or replacing $A$ by an integral extension, we may assume that $B$ is a field and $A$ is integrally closed in $B$. Then, by Lemma $1, A$ is completely integrally closed.

Let $c_{1}, \ldots, c_{n^{2}} \in \Gamma$ be a basis for $M_{n}(B)$ as a vector space over $B$. Take any $\gamma \in A(\Gamma)$, and write $\gamma=\sum b_{i} c_{l}$. Then, for each $j$,

$$
\operatorname{tr}\left(\gamma c_{j}\right)=\sum_{i} b_{i} \operatorname{tr}\left(c_{i} c_{J}\right) \text {. }
$$

By hypothesis, all the traces appearing in (2) lie in $A$. Viewing (2) as $n^{2}$ equations in the variables $b_{1}, \ldots, b_{n^{2}}$, it follows by Cramer's rule that $\delta b_{i} \in A, i=1, \ldots, n^{2}$, where $\delta=\operatorname{det}\left(\operatorname{tr}\left(c_{l} c_{J}\right)\right) \in A$. By the nondegeneracy of the trace, $\delta \neq 0$. Let $T=\sum_{i=1}^{n^{2}} A\left(\delta c_{i}\right)$; since $\delta b_{i} \in A$, we have

$$
\delta^{2} A(\Gamma) \subseteq T \text {. }
$$

To see that $T$ is actually a ring we make a similar computation. Let

$$
c_{i} c_{j}=\sum_{k} \beta_{i j k} c_{k} \text {. }
$$

Multiplying (4) by any $c_{l}$ and taking traces, we have

$$
\operatorname{tr}\left(c_{i} c_{j} c_{l}\right)=\sum_{k} \beta_{l j k} \operatorname{tr}\left(c_{k} c_{l}\right) \text {. }
$$

Again, the traces in (5) lie in $A$, so (fixing $i$ or $j$ ) by Cramer's rule $\delta \beta_{i j k} \in A$. Thus, rewriting (4) as

$$
\left(\delta c_{i}\right)\left(\delta c_{j}\right)=\sum_{k}\left(\delta \beta_{i j k}\right)\left(\delta c_{k}\right)
$$

we see that $T$ is closed under multiplication. Since $T$ is also a finitely generated $A$-module, it is integral over $A$. Therefore, Lemma 2 and (3) above show that $A(\Gamma)$ is integral over $A$. This contradiction completes the proof. 
COROLlARY 7 (Bass). If, in addition to the hypotheses at the beginning of the section, $A$ is Noetherian and $\Gamma$ is a finitely generated monoid, then $A(\Gamma)$ is a finitely generated A-module.

Proof. As noted earlier, the Mori-Nagata theorem assures that the integral closure of a Noetherian domain is c.i.c. Therefore, by Prop. 6, $A(\Gamma)$ is integral over $A$. Since, in addition, $A$ is Noetherian and $A(\Gamma)$ is a finitely generated p.i. $A$-algebra, it follows by a theorem of Procesi $[\mathbf{P}, \mathrm{p}$. $128]$ that $A(\Gamma)$ is a finitely generated $A$-module.

EXAMPLE 8. Let $F$ be any field of prime characteristic $p$, and let $x$ and $y$ be commuting indeterminates over $F$. Let $C=F[x, y] ; J=y C, A$ the subring $F+J$ of $C$, and $B$ the quotient field of $C$. In the matrix ring $M_{p}(B)$, let $I$ be the identity matrix, and $\left\{E_{i j}\right\}$ the usual matrix units. Let $\Gamma$ be the monoid generated by $\left\{x I+y E_{i j} \mid 1 \leq i, j \leq p\right\}$. Then $B(\Gamma)=$ $M_{p}(B)$, and for each $\gamma \in \Gamma, \operatorname{tr}(\gamma) \in A$. But none of the generators of $\Gamma$ is integral over $A$. So, $(*)$ does not hold.

Proof. The $p^{2}$ generators of $\Gamma$ are linearly independent over $B$; hence, $B(\Gamma)=M_{p}(B)$. Note that $\Gamma \subseteq M_{p}(C)$, and in $M_{p}(C / J)$ the image of $\Gamma$ is generated by scalar matrices; so the image must consist entirely of scalar matrices, which have trace 0 . Thus, for $\gamma \in \Gamma, \operatorname{tr}(\gamma) \in J \subseteq A$. However, $x I+y E_{i j}$ cannot be integral over $A$, since its image in $M_{p}(C / J)$ is clearly not integral over $A / J \cong F$.

Remarks. Example 8 shows the need for the hypotheses in Prop. 5 and Prop. 6. In the example $A$ is integrally closed, but its complete integral closure is $C$. By slightly modifying the example, one can obtains a counterexample to $(*)$ for any $n \geq p$ and any $\operatorname{ring} A$ with a prime ideal $P$ such that $A / P$ has characteristic $p$, and the integral closure of $A / P$ is not c.i.c. E.g., to obtain a counterexample in characteristic 0 , replace $F$ in Ex. 8 by the ring $\mathbf{Z}$ of integers, and $J$ by the ideal of $\mathbf{Z}[x, y]$ generated by $p$ and $y$.

\section{REFERENCES}

[A] S. A. Amitsur, Identities and linear dependence, Israel J. Math., 22 (1975), 127-137.

[AT] E. Artin and J. T. Tate, A note on finite ring extensions, J. Math. Soc. Japan, 3 (1951), 74-77.

[B] H. Bass, Groups of integral representation type, Pacific J. Math., 86 (1980), 15-51.

[Bo] N. Bourbaki, Algèbre Commutative, Ch. 7, Hermann, Paris, 1965. 
$\left[\mathrm{Br}_{1}\right]$ A. Braun, Affine polynomial identity rings and their generalizations, J. Algebra, 58 (1979), 481-494.

$\left[\mathrm{Br}_{2}\right] \longrightarrow$ A characterizations of prime Noetherian p.i. rings and a theorem of MoriNagata, Proc. Amer. Math. Soc., 74 (1979), 9-15.

[C] G. Chrystal, Textbook of Algebra, I, 7th Ed., Chelsea, New York, 1964.

[G] R. Gilmer, Multiplicative Ideal Theory, Queen's Papers in Pure and Applied Math., No. 12, Queen's University, Kingston, Ontario, 1968.

[H] I. N. Herstein, Topics in Algebra, 2nd Ed., Xerox, Lexington, Mass., 1975.

[K] W. Krull, Beiträge zur Arithmetik kommutativer Integritätsbereiche, II, Math. Z., 41 (1936), 665-679.

[N] M. Nagata, Local Rings, Interscience, New York, 1962.

[Nk] T. Nakayama, On Krull's conjecture concerning completely integrally closed integrity domains, I, II, Proc. Imp. Acad. Tokyo, 18 (1942), 185-187, 233-236; III, Proc. Japan Academy, 22 (1946), 249-250.

[P] C. Procesi, Rings with Polynomial Identity, Marcel Dekker, New York, 1973.

[S] W. Schelter, Integral extensions of rings satisfying a polynomial identity, J. Algebra, 40 (1976), 245-257.

Received June 2, 1983. Supported in part by the National Science Foundation.

University of California at San Diego

LA Jolla, CA 92093 


\section{PACIFIC JOURNAL OF MATHEMATICS \\ EDITORS}

Donald BABBITT (Managing Editor)

University of California

Los Angeles, CA 90024

J. Dugundu

University of Southern Californa

Los Angeles, CA 90089-1113

R. FINN

Stanford University

Stanford, CA 94305

HermanN FLaSChKa

University of Arizona

Tucson, AZ 85721

\author{
C. C. MOORE \\ University of California \\ Berkeley, CA 94720 \\ ARTHur Ogus \\ University of California \\ Berkeley, CA 94720 \\ Hugo Rossi \\ University of Utah \\ Salt Lake City, UT 84112 \\ H. SAMELSON \\ Stanford University \\ Stanford, CA 94305
}

ASSOCIATE EDITORS
R. ARENS
E. F. BECKENBACH
B. H. NeUmanN
F. WOLF
K. YOSHIDA (1906-1982)

\section{SUPPORTING INSTITUTIONS}

UNIVERSITY OF ARIZONA

UNIVERSITY OF BRITISH COLUMBIA

UNIVERSITY OF OREGON

CALIFORNIA INSTITUTE OF TECHNOLOGY

UNIVERSITY OF CALIFORNIA

MONTANA STATE UNIVERSITY

UNIVERSITY OF SOUTHERN CALIFORNIA

UNIVERSITY OF NEVADA, RENO

STANFORD UNIVERSITY

UNIVERSITY OF HAWAII

NEW MEXICO STATE UNIVERSITY

UNIVERSITY OF TOKYO

UNIVERSITY OF UTAH

WASHINGTON STATE UNIVERSITY

OREGON STATE UNIVERSITY

UNIVERSITY OF WASHINGTON 


\section{Pacific Journal of Mathematics}

\section{Vol. 116, No. $1 \quad$ November, 1985}

K. Adachi, Le problème de Lévi pour les fibrés grassmanniens et les variétés

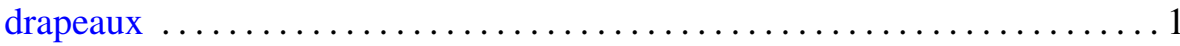

John MacLeod Ball, Remarks on the paper: "Basic calculus of variations" . . . 7 John Kelly Beem and Phillip E. Parker, Whitney stability of solvability . . . 11 Alberto Facchini, Decompositions of algebraically compact modules .......25

S. S. Khare, Finite group action and equivariant bordism $\ldots \ldots \ldots \ldots . \ldots 39$

Horst Leptin, A new kind of eigenfunction expansions on groups $\ldots \ldots \ldots . .45$

Pei-Kee Lin, Unconditional bases and fixed points of nonexpansive

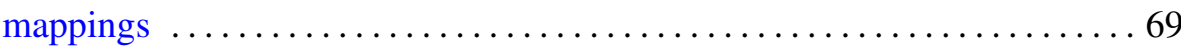

Charles Livingston, Stably irreducible surfaces in $S^{4} \ldots \ldots \ldots \ldots \ldots 77$

Kevin Mor McCrimmon, Nonassociative algebras with scalar involution . . .885

Albert Milani, Singular limits of quasilinear hyperbolic systems in a

bounded domain of $\mathbf{R}^{3}$ with applications to Maxwell's equations

Takemi Mizokami, On $M$-structures and strongly regularly stratifiable

spaces

Jesper M. Møller, On the homology of spaces of sections of complex

projective bundles

Nikolaos S. Papageorgiou, Carathéodory convex integrand operators and

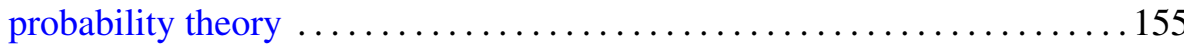

Robert John Piacenza, Transfer in generalized prestack cohomology 185

Lance W. Small and Adrian R. Wadsworth, Integrality of subrings of matrix rings ...

James Michael Wilson, On the atomic decomposition for Hardy spaces 